\title{
Postmortem Brain Tissue of Depressed Suicides Reveals Increased Gs $\alpha$ Localization in Lipid Raft Domains Where It Is Less Likely to Activate Adenylyl Cyclase
}

\author{
Robert J. Donati, ${ }^{1,3}$ Yogesh Dwivedi, ${ }^{2}$ Rosalinda C. Roberts, ${ }^{4}$ Robert R. Conley, ${ }^{4}$ Ghanshyam N. Pandey, ${ }^{2}$ and \\ Mark M. Rasenick ${ }^{1,2}$ \\ Departments of ${ }^{1}$ Physiology and Biophysics and ${ }^{2}$ The Psychiatric Institute, University of Illinois at Chicago, College of Medicine, Chicago, Illinois 60612 - \\ 7342, ${ }^{3}$ Basic and Health Science Department, Illinois College of Optometry, Chicago, Illinois 60616, and ${ }^{4}$ Maryland Psychiatric Research Center, Baltimore, \\ Maryland 21228
}

Recent in vivo and in vitro studies have demonstrated that Gs $\alpha$ migrates from a Triton X-100 (TX-100)-insoluble membrane domain (lipid raft) to a TX-100-soluble nonraft membrane domain in response to chronic, but not acute, treatment with tricyclic or selective serotonin reuptake inhibitor antidepressants. This migration resulted in a more facile association with adenylyl cyclase. Our hypothesis is that $\mathrm{Gs} \alpha$ may be ensconced, to a greater extent, in lipid rafts during depression, and that one action of chronic antidepressant treatment is to reverse this. In this postmortem study, we examined Gs $\alpha$ membrane localization in the cerebellum and prefrontal cortex of brains from nonpsychiatric control subjects and suicide cases with confirmed unipolar depression. Sequential TX-100 and TX-114 detergent extractions were performed on the brain tissue. In the cerebellum, the ratio of TX-100/TX-114-soluble Gs $\alpha$ is $\sim 2: 1$ for control versus depressed suicides. Results with prefrontal cortex samples from each group demonstrate a similar trend. These data suggest that depression localizes Gs $\alpha$ to a membrane domain (lipid rafts) where it is less likely to couple to adenylyl cyclase and that antidepressants may upregulate Gs $\alpha$ signaling via disruption of membrane microenvironments. Raft localization of Gs $\alpha$ in human peripheral tissue may thus serve as a biomarker for depression and as a harbinger of antidepressant responsiveness.

Key words: antidepressant; depression; lipid microdomains; cerebellum; prefrontal cortex; signal transduction

\section{Introduction}

Despite decades of research, the molecular and cellular implications of chronic antidepressant treatment remain unknown, as does the target of this treatment in the human brain. Imaging studies suggest that many regions of the human brain are involved in depression (Drevets, 2001; Mayberg, 2003) and it has been proposed that elements of the hypothalamic/pituitary/adrenocortical axis are involved in the ontogeny of depression and are important targets of antidepressant therapy (Meltzer et al., 1982; Valentino and Curtis, 1991; Owens and Nemeroff, 1999; Holsboer, 2000). Nonetheless, to act, antidepressants are likely to

\footnotetext{
Received April 20, 2007; revised Jan. 28, 2008; accepted Jan. 30, 2008.

This work was supported by U.S. Public Health Service Grants MH 39595 and DA020568 (M.M.R.), R01 MH 48153 (G.N.P.), MH 01836 and MH 068777 (Y.D.), and R01 MH60744 and MH 60744 (R.C.R.), and by a Distinguished Investigator grant (G.N.P.) and a Standard Research grant (Y.D.) from the American Foundation for Suicide Prevention (New York, NY). We thank Barbara Brown and Miljana Petkovic for their help in organizing the brain tissue and Robyn Tamboli and Justin Brown for technical advice and assistance, respectively. We thank Robert Gibbons for help with statistical analysis. We also thank the members of the Maryland Brain Collection for their efforts, particularly in family interviews and dissection. Weare grateful for the cooperation of Office of the Chief Medical Examiner (Baltimore, MD).

Correspondence should be addressed to Dr. Mark M. Rasenick, Department of Physiology and Biophysics, University of Illinois at Chicago, College of Medicine, 835 South Wolcott Avenue, M/C 901, Room E202, Chicago, IL 60612-7342.E-mail: raz@uic.edu.

R. C. Roberts' present address: Department of Psychiatry and Behavioral Neurobiology, University of Alabama, School of Medicine, Birmingham, AL 35294.

R. R. Conley's present address: Department of Neuroscience, Eli Lilly, Indianapolis, IN 46285.

DOI:10.1523/JNEUROSCI.5713-07.2008

Copyright $\odot 2008$ Society for Neuroscience $\quad$ 0270-6474/08/283042-09\$15.00/0
}

have one or more primary molecular targets. Some of these targets are monoamine uptake sites, but it is difficult to reconcile the clinical requirement for chronic drug or electroconvulsive shock treatment with uptake inhibition, which is contemporaneous with acute drug exposure. Antagonism of $5-\mathrm{HT}_{3}$ receptor activation of $\mathrm{Na}^{+}$and $\mathrm{Ca}^{2+}$ currents has recently been proposed to be a result of antidepressant action (Eisensamer et al., 2003). Although other targets for antidepressant drugs have not been precisely identified, they may be at or near the membrane, and altered intracellular signaling is often among the initial effects of antidepressant treatment. More specifically, the various mechanisms proposed for chronic antidepressant action are consistent with a long-term increase in cAMP production (Donati and $\mathrm{Ra}$ senick, 2003).

In 1983, we first reported that long-term administration of various antidepressants enhanced guanylyl-5' - -imidodiphoshate [Gpp(NH)p]- and fluoride-stimulated adenylyl cyclase activity in rat cortex and hypothalamus membranes (Menkes et al., 1983). This suggested that the stimulatory $\alpha$-subunit of the G-protein, Gs, was at least an indirect target of antidepressant action and that antidepressant treatment facilitated the activation of adenylyl cyclase by Gs. These initial findings have been substantiated by later in vivo and in vitro studies (Ozawa and Rasenick, 1989, 1991; Perez et al., 1989, 1991; De Montis et al., 1990; Chen and Rasenick, 1995; Kamada et al., 1999). 
The relationship between neurotransmitter signaling and lipid raft nanodomains has become an increasingly important area of research in neuroscience (for review, see Allen et al., 2007). In vitro studies using C6 glioma cells and in vivo studies with rat brain membranes demonstrate that Gs $\alpha$ migrates from a Triton X-100 (TX-100)-resistant lipid raft containing membrane domain to a TX-100-soluble non-lipid raft membrane domain in response to chronic antidepressant treatment (Toki et al., 1999; Donati and Rasenick, 2005). Additional evidence suggests Gs $\alpha$ as a preferential target for antidepressant action (Toki et al., 1999; Donati and Rasenick, 2005). Interestingly, antidepressant and antipsychotic drugs have been shown to concentrate in raft-like domains in both HEK293 and NIE-115 neuroblastoma cells (Eisensamer et al., 2005). Together, these studies suggest that the lipid environment of the G-protein may play an important role in its localization and function, and that chronic antidepressant treatment alters the membrane localization of $\mathrm{Gs} \alpha$, resulting in augmented coupling to adenylyl cyclase.

The current study examines the membrane localization of Gs $\alpha$ in postmortem human brain of depressed suicide subjects and nonsuicide controls without known psychiatric histories. Sequential detergent extraction of both cerebellum and prefrontal cortex (PFC) was performed to determine the relative amount of Gs $\alpha$ in TX-100-soluble non-lipid raft domains (TX-100 extract) versus TX-100-resistant lipid raft/cytoskeleton-associated membrane domains (TX-114 extract) in these brain regions. There was a significant amount of Gs $\alpha$ localized to TX-100-resistant membrane domains in the suicide subjects compared with the nonsuicide controls as determined by the ratio of TX-100- to TX-114-extractable Gs $\alpha$. These data suggest that Gs $\alpha$ is less available for adenylyl cyclase signaling in the depressed brain and is consistent with the observation that a therapeutic effect of antidepressants may be to move Gs $\alpha$ out of cytoskeletal-associated lipid raft-like domains into a membrane compartment where it is more available to interact with adenylyl cyclase. Both these postmortem data and previous studies in model systems suggest that membrane microdomains are involved in both depression and therapies for that disease. They also suggest that the degree of raft localization of Gs $\alpha$ might serve as a biomarker for human depression and antidepressant response.

\section{Materials and Methods}

Human subject information. The study was performed in Brodmann's area 9 and cerebellum obtained from the right hemisphere of suicide subjects $(n=16)$ and normal control subjects $(n=16)$. Tissues were obtained from the Maryland Brain Collection at the Maryland Psychiatric Research Center (Baltimore, MD).

All tissues from normal controls and suicide subjects were screened for evidence of neuropathology. In addition, in each case, screening for the presence of HIV was done in blood samples, and all HIV-positive cases were excluded. Toxicology data were obtained by the analysis of urine and blood samples. $\mathrm{pH}$ of the brain was measured in cerebellum in all cases as described by Harrison et al. (1995). Psychiatric drugs in common use as well as drugs of abuse were screened for by using mass spectroscopy. Prescribed drugs were also screened for in interviews.

We excluded normal control subjects who had a known psychiatric illness or had a history of alcohol or substance of abuse disorder. However, comorbidity, particularly, alcohol or substance of abuse, is often present with other psychiatric illnesses in suicide subjects.

Diagnostic method. Families were queried on all medications or drugs of abuse by trained interviewers. At least one family member, after giving written informed consent, underwent an interview based on the Diagnostic Evaluation After Death (DEAD) (Zalcman and Endicott, 1983) and the Structured Clinical Interview for the DSM-IV (SCID) (Spitzer et al., 1992). The interviews were done by a trained psychiatric social worker. Two psychiatrists independently reviewed the write-up from this interview, as well as the SCID that was completed from it, as part of their diagnostic assessment of the case. Diagnoses were made from the data obtained in this interview, medical records from the case, and records obtained from the Medical Examiner's office. The two diagnoses were compared and discrepancies were resolved by means of a consensus conference. Controls were verified as free from mental illnesses using these consensus diagnostic procedures. This study was approved by the institutional review boards of the University of Illinois at Chicago and the University of Maryland, Baltimore.

Sequential detergent extraction of brain membranes. Brain samples were dissected from the fresh brain and stored at $-80^{\circ} \mathrm{C}$ or dissected from frozen brain tissue with a Stryker autopsy saw, repackaged, and stored at $-80^{\circ} \mathrm{C}$ until use. Because of the small amount of tissue available for these experiments, traditional sucrose density raft membrane preparations were not used. Instead, a sequential detergent extraction procedure previously used by Toki et al. (1999) was modified to differentiate between raft-localized and non-raft-localized Gs $\alpha$. Brain samples (cerebellum or PFC) were resuspended and minced in TME buffer (10 mM Tris- $\mathrm{HCl}, 1$ mм $\mathrm{MgCl}_{2}, 1$ mм EDTA, pH 7.5; $\sim 1 \mathrm{ml} / 100 \mathrm{mg}$ tissue) followed by homogenization in a motorized Teflon glass homogenizer. The samples were centrifuged at $100,000 \times g$ for $1 \mathrm{~h}$ at $4^{\circ} \mathrm{C}$, and the supernatant (cytosol) and pellet (membrane) were saved. The crude membrane pellet was extracted with $0.75 \mathrm{ml}$ of TME containing $1 \%$ Triton X-100 for $1 \mathrm{~h}$ at $4^{\circ} \mathrm{C}$ followed by homogenization as above. This sample was centrifuged as above and both the supernatant (TX-100 extract) and pellet (TX-100resistant membrane fraction) were saved. This pellet was extracted with $0.75 \mathrm{ml}$ of TME containing $1 \%$ Triton X-114 for $1 \mathrm{~h}$ at $4^{\circ} \mathrm{C}$ and homogenized as above. The sample was centrifuged as above and both the supernatant (TX-114 extract) and pellet (detergent-insoluble pellet) were saved. The detergent-insoluble pellet could not be efficiently solubilized to be quantified. From here on out, the TX-100 extract will be referred to as the TX-100-soluble domain and the TX-114 extract will be referred to as the TX-100-resistant domain. All fractions were assayed for protein content (Bio-Rad Protein Assay; Bio-Rad, Hercules, CA) and frozen at $-20^{\circ} \mathrm{C}$ until further use. Frontal cortex and cerebellum were the only brain regions available for these experiments.

SDS-PAGE and Western blotting. TX-100- and TX-114-soluble (TX100 -resistant) membrane fractions $(12-15 \mu \mathrm{g})$ were analyzed by SDSPAGE followed by Western blotting. The gels were transferred to polyvinylidene difluoride membranes (Immobilon-P; Millipore, Bedford, MA) by Western blotting and probed with an anti-Gs $\alpha$ antibody (DuPont NEN, Boston, MA; Calbiochem, La Jolla, CA) or anti-Gq $\alpha$ antibody (Santa Cruz Biotechnology, Santa Cruz, CA) followed by secondary antibody [HRP-linked anti-rabbit IgG $\mathrm{F}\left(\mathrm{ab}^{\prime}\right)_{2}$ from Jackson ImmunoResearch (West Grove, PA)]. Some of the blots probed with the Gs $\alpha$ antibody were stripped with $100 \mathrm{~mm}$ glycine, $\mathrm{pH} 2.3$, before being probed with the Gq $\alpha$ antibody. Blots were incubated with the chemiluminescent reagent ECL (Amersham Biosciences, Piscataway, NJ) and exposed to $\mathrm{x}$-ray film. There were an equal number of control and suicide TX-100 and TX-114 samples per autoradiogram. The autoradiograms were quantitated by densitometry using ImageQuant software (Molecular Dynamics, Sunnyvale, CA) and the ratio of TX-100-soluble nonraft G-protein (TX-100) to TX-100-resistant G-protein (TX-114) was compared. Additionally, the total amounts of detergent extracted Gs $\alpha$ (TX100 plus TX-114) were compared as above.

Normalization. To be consistent throughout the data collection, the TX-100/TX-114 ratio for each sample was normalized to the mean TX100/TX-114 ratio of all of the samples (control and suicide) run on that particular autoradiogram (normalized ratio $=$ sample ratio $/$ mean ratio). Additionally, this normalization procedure was repeated when comparing the amount of Gs $\alpha$ extracted with each detergent (normalized densitometry value $=$ sample value/mean value). This allowed us to compare samples accurately among gels and their corresponding autoradiograms.

Statistical methods. Gel and Western blot data were analyzed for statistical significance by unpaired, two-tailed Student's $t$ test or one-way ANOVA using Prism 4.0 software package for statistical data analysis (Graph Pad, San Diego, CA). Means are \pm SEM, and differences for all experiments were considered significant at $p<0.05\left({ }^{*} p<0.05\right.$; ${ }^{* *} p<$ 
Table 1. Demographic characteristics of suicide and control subjects

\begin{tabular}{|c|c|c|c|c|c|c|c|c|}
\hline & & $\begin{array}{l}\text { Age } \\
\text { (years) }\end{array}$ & Race & Gender & PMI (h) & Cause of death & $\begin{array}{l}\text { Drug screen } \\
\text { (at the time of death) }\end{array}$ & Psychiatric diagnosis \\
\hline 1 & Control & 22 & Black & Male & 19 & GSW & None & Normal \\
\hline 2 & Control & 37 & Black & Male & 5 & ASCVD & None & Normal \\
\hline 3 & Control & 31 & Black & Male & 8 & GSW & Lidocaine & Normal \\
\hline 4 & Control & 46 & Black & Male & 9 & Multiple injuries & None & Normal \\
\hline 5 & Control & 33 & White & Male & 15 & GSW & Acetaminophen & Normal \\
\hline 6 & Control & 48 & White & Male & 26 & ASCVD & None & Normal \\
\hline 7 & Control & 40 & White & Female & 7 & ASCVD & None & Normal \\
\hline 8 & Control & 23 & Black & Male & 15 & GSW & None & Normal \\
\hline 9 & Control & 38 & Black & Male & 16 & Lung sarcoidosis & None & Normal \\
\hline 10 & Control & 83 & White & Male & 20 & ASCVD & None & Normal \\
\hline 11 & Control & 65 & Black & Female & 23 & ASCVD & None & Normal \\
\hline 12 & Control & 35 & White & Male & 24 & Crush injury to abdomen and chest & None & Normal \\
\hline 13 & Control & 52 & White & Male & 30 & ASCVD & None & Normal \\
\hline 14 & Control & 63 & White & Female & 30 & Ovarian cancer & None & Normal \\
\hline 15 & Control & 37 & White & Male & 24 & ASCVD & None & Normal \\
\hline 16 & Control & 45 & White & Male & 22 & ASCVD & None & Normal \\
\hline 1 & Suicide & 26 & Black & Male & NA & Hanging & Cocaine & NA \\
\hline 2 & Suicide & 21 & Black & Male & 4 & Hanging & Cocaine, morphine & Opiate abuse, cocaine abuse \\
\hline 3 & Suicide & 50 & White & Male & 7 & GSW & None & No psychiatric illness \\
\hline 4 & Suicide & 27 & White & Male & 24 & GSW & None & Major depression, alcohol abuse \\
\hline 5 & Suicide & 24 & White & Male & 7 & GSW & Ethanol & Major depression \\
\hline 6 & Suicide & 21 & White & Male & 17 & GSW & None & $\begin{array}{l}\text { Adjustment disorder with mixed disturbance of } \\
\text { emotions and conduct }\end{array}$ \\
\hline 7 & Suicide & 75 & White & Male & 18 & GSW & None & Adjustment disorder/conduct disorder \\
\hline 8 & Suicide & 41 & Black & Male & 12 & $\begin{array}{l}\text { Jumping } \\
\text { suicide }\end{array}$ & None & Not enough information \\
\hline 9 & Suicide & 41 & White & Female & 27 & Drug overdose & $\begin{array}{l}\text { Amitriptyline, desipramine, diphenhydramine, } \\
\text { nortriptyline, pseudophedrine, salicylate, ethanol }\end{array}$ & Major depression, alcohol abuse \\
\hline 10 & Suicide & 68 & White & Female & 26 & GSW & Amitriptyline & Bipolar disorder \\
\hline 11 & Suicide & 22 & Black & Female & 16 & Drug overdose & Propranolol & Major depression \\
\hline 12 & Suicide & 39 & White & Male & 30 & Asphyxia & Freon, cocaine, and metabolites & Cocaine abuse \\
\hline 13 & Suicide & 30 & White & Male & 32 & Hanging & Cocaine, ethanol & Alcohol abuse, cocaine abuse, drug abuse \\
\hline 14 & Suicide & 46 & White & Female & 21 & Drug overdose & Amitriptyline, desipramine, ranitidine, ethanol & Major depression \\
\hline 15 & Suicide & 20 & White & Male & 32 & Hanging & Ethanol & Alcohol abuse \\
\hline 16 & Suicide & 21 & White & Male & 23 & Hanging & None & Adjustment disorder conduct \\
\hline
\end{tabular}

ASCVD, Atherosclerotic cardiovascular disease; GSW, gunshot wound; NA, not available.

0.02). The differences in TX-100 and TX-114 Gs $\alpha$, age, gender, $\mathrm{pH}$ of the brain, and postmortem interval (PMI) between depressed and control subjects were analyzed using the independent-sample $t$ test. The relationships between TX-100 and TX-114 Gs $\alpha$ and PMI, and age were determined by Pearson product-moment correlation analysis. Values of $p$ were two-tailed. During data analysis, confounding variables such as age, $\mathrm{PMI}$, gender, and $\mathrm{pH}$ of the brain were also used as covariates (ANCOVA). The effect of the presence of antidepressant drugs in serum was evaluated in normal controls and suicide subjects using antidepressant toxicology screens as covariate (ANCOVA). In addition, effect of antidepressant was also evaluated by comparing depressed subjects with positive antidepressant screening results $(n=3)$, and depressed subjects not revealing antidepressants in toxicology screens.

\section{Results}

There were 13 males and 3 females in the control group, and 12 males and 4 females in the suicide group. The age range was 21-87 years, whereas the PMI was in the range of 5-32 h (Table $1)$. There were no significant differences in age $(t=0.018$; $\mathrm{df}=$ $27 ; p=0.86)$ or PMI $(t=1.01$; $\mathrm{df}=26 ; p=0.29)$ between suicides and normal control subjects. The mean brain $\mathrm{pH}$ values of normal controls and suicides were $6.07 \pm 0.4$ and $6.28 \pm 0.36$, respectively, which were not different between these groups $(t=$ $1.4 ; \mathrm{df}=27 ; p=0.16)$.
Gs $\alpha$ is distributed in both TX-100-resistant lipid raft/ cytoskeleton-associated and TX-100-soluble non-lipid raft membrane domains of human cerebellum and prefrontal cortex

Membranes from tissue samples were prepared as described in Materials and Methods. SDS-PAGE and Western blotting revealed abundant membrane-associated Gs $\alpha$ in both the cerebellum (Fig. 1A) and the PFC (Fig. 1B) of depressed suicide and control subjects. Additionally, Gs $\alpha$ localized to both TX-100resistant membrane domains (Fig. 1, TX-114) and TX-100soluble membrane domains (Fig. 1, TX-100) and was detected in both brain regions in control and suicide subjects.

Depressed subjects have a greater proportion of Gs $\alpha$ localized in TX-100-resistant membrane domains

There is conflicting evidence as to whether there is an alteration in the amount of Gs $\alpha$ found in various brain regions of individuals with major depression. It has been demonstrated that there is an increase in the amount of Gs $\alpha$ protein (Pacheco et al., 1996) and mRNA (Dwivedi et al., 2002) in the frontal cortex of subjects with major depression, but there are also reports showing no change in the amount of Gs $\alpha$ protein (Dowlatshahi et al., 1999). In this experiment, the total amount of TX-100- and TX-114- 
A.

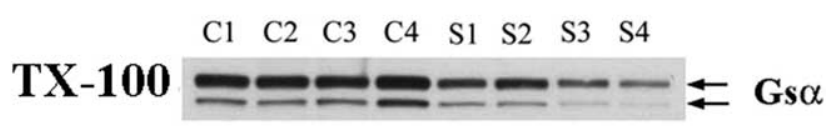

TX-114

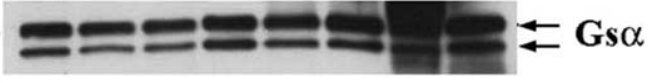

B.

\section{CONTROL}
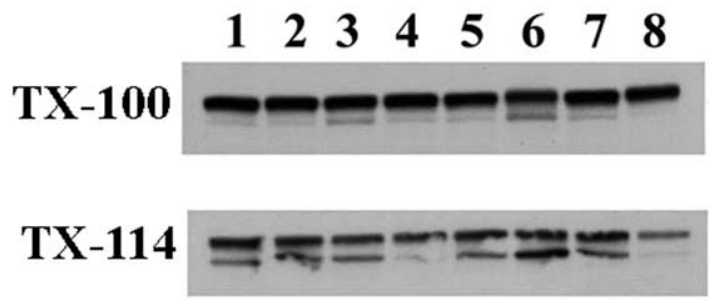

SUICIDE
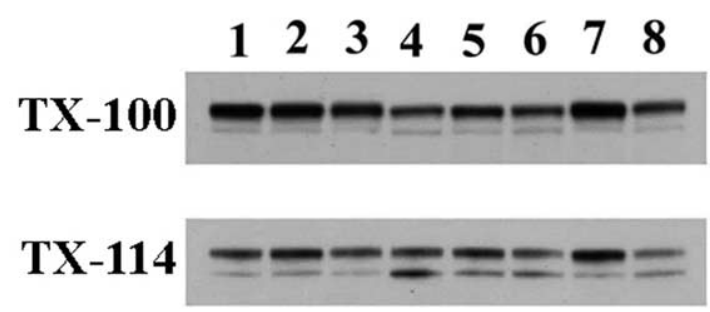

Figure 1. Representative SDS-PAGE gel autoradiograms for cerebellum and prefrontal cortex samples. $A$, Representative $G s \alpha$ immunoblots of TX-100 and TX-114 extracts from cerebellum membranes of control (C) and depressed suicide (S) subjects. $\boldsymbol{B}$, Representative Gs $\alpha$ immunoblots of TX-100 and TX-114 extracts from prefrontal cortex membranes of control and depressed suicide subjects. Gs $\alpha$ bands are indicated by arrows.

extractable Gs $\alpha$ in cerebellum membranes was unchanged between control and suicide subjects [control $(n=14)$ : mean, $0.9547 \pm 0.2759 \mathrm{AU}$; suicide $(n=13)$ : mean, $1.049 \pm 0.3959 \mathrm{AU}$; $p=0.4775]$. However, there was a small but significant difference in the total detergent-extractable Gs $\alpha$ (TX-100 plus TX-114) in the PFC between the two groups [control $(n=14)$ : mean, $1.188 \pm 0.2027 \mathrm{AU}$; suicide $(n=14)$ : mean, $0.8137 \pm 0.2670 ; p=$ $0.003]$. This is an indication that there is a greater fraction of detergent-insoluble Gs $\alpha$ in the PFC of suicide subjects. Two different extractable membrane domains, TX-100-resistant (TX114) and TX-100-soluble (TX-100) were examined by ANCOVA using age, $\mathrm{PMI}$, gender, or $\mathrm{pH}$ of the brain separately as covariate. A significantly lower amount of non-raft TX-100-soluble Gs $\alpha$ was found in the PFC of suicide subjects compared with the same region in control subjects when age $(\mathrm{df}=1,26 ; F=21.71 ; p<$ $0.001)$, gender $(\mathrm{df}=1,26 ; F=21.77 ; p<0.001)$, $\mathrm{PMI}(\mathrm{df}=1,26$; $F=22.76 ; p<0.001)$, or $\mathrm{pH}$ of the brain $(\mathrm{df}=1,26 ; F=19.04$; $p<0.001$ ) were used as covariate during ANCOVA analysis (Fig. $2 B)$. The decrease in this pool of Gs $\alpha$ accounts for the majority of the decrease of total detergent-extracted Gs $\alpha$ in the suicide subjects, although there is a decrease in TX-100-resistant Gs $\alpha$ as well. For example, TX-114 level was decreased in suicide subjects when the data were analyzed by ANCOVA using age $(\mathrm{df}=1,26 ; F=$
A.

\section{Cerebellum}

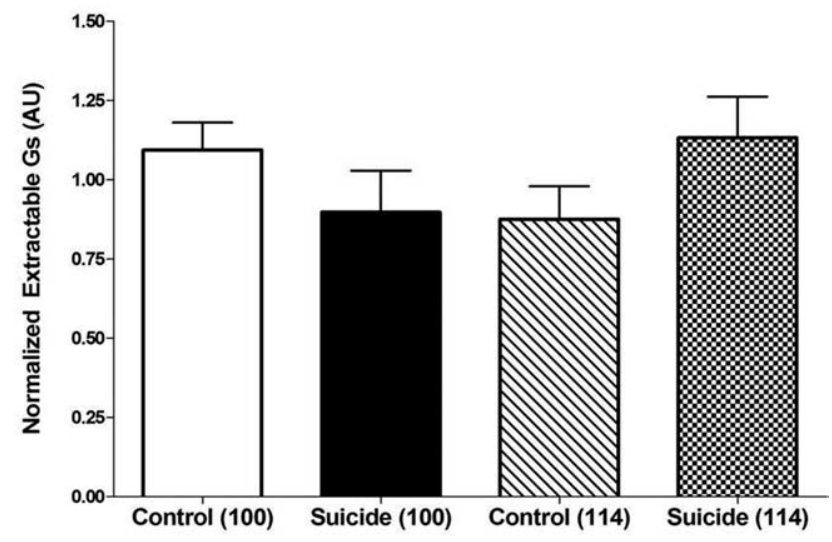

B.

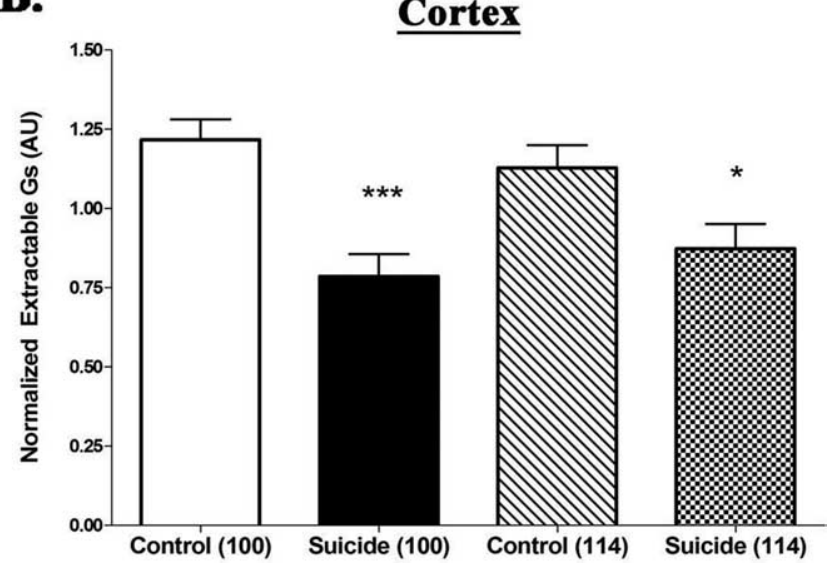

Figure 2. Comparison of the total extractable Gs $\alpha$ in both the cerebellum and prefrontal cortex of depressed and control subjects. Graphic representation of the normalized densitometric values of TX-100-soluble (100) and TX-100-resistant (114) Gs $\alpha$ from both cerebellum and prefrontal cortex. A, Data were acquired from densitometric determinations of gels and autoradiographs on cerebellum samples. Control versus suicide TX-100-soluble (100) and control versus suicide TX-100-resistant (114) Gs $\alpha$ were examined. A one-way ANOVA followed by a Newman-Keuls multiple-comparison test was performed and the means were not found to be significantly different $(p>0.05)$. There was no significant difference in the total amount (TX-100 plus TX-114) of extractable Gs $\alpha$ ( $n=14$ for control and $n=13$ for suicide subjects). $\boldsymbol{B}$, Data were acquired from densitometric determinations of gels and autoradiographs from prefrontal cortex samples. Control versus suicide TX-100-soluble (100) and control versus suicide TX-100-resistant (114) Gs $\alpha$ were examined. A one-way ANOVA followed by a Newman-Keuls multiple-comparison test was performed, and the means were found to be significantly different. ${ }^{* * *} p<0.001$, control versus suicide TX-100. ${ }^{*} p<0.05$, control versus suicide TX-114. There was a significant difference in the total amount (TX-100 plus TX-114) of extractable Gs $\alpha$ in control versus suicide subjects, $p<0.0003$ ( $n=14$ for control and $n=14$ for suicide subjects), in the prefrontal cortex, but not in the cerebellum. Error bars indicate SEM.

6.03; $p=0.02)$, gender $(\mathrm{df}=1,26 ; F=6.26 ; p=0.019)$, PMI $(\mathrm{df}=1,26 ; F=5.84 ; p=0.02)$, or $\mathrm{pH}$ of the brain $(\mathrm{df}=1,26 ; F=$ 5.96; $p=0.022)$. Similarly, the levels of individual TX-100 ( $\mathrm{df}=$ $1,26 ; F=17.8 ; p<0.001)$ and TX-114 $(\mathrm{df}=1,26 ; F=4.2 ; p=$ 0.04 ) remained significantly different between normal controls and suicide subjects in PFC whether or not antidepressants were present in the toxicology screens.

Furthermore, when antidepressant toxicology was factored in by ANCOVA, there was still no significant difference in the TX100-soluble or TX-100-resistant Gs $\alpha$ extracted in the cerebellum between control and suicide subjects (Fig. 2A). Although the total amount of detergent-extractable Gs $\alpha$ differs in the PFC between control and suicide subjects (Fig. 2B), there is a similar 
A.

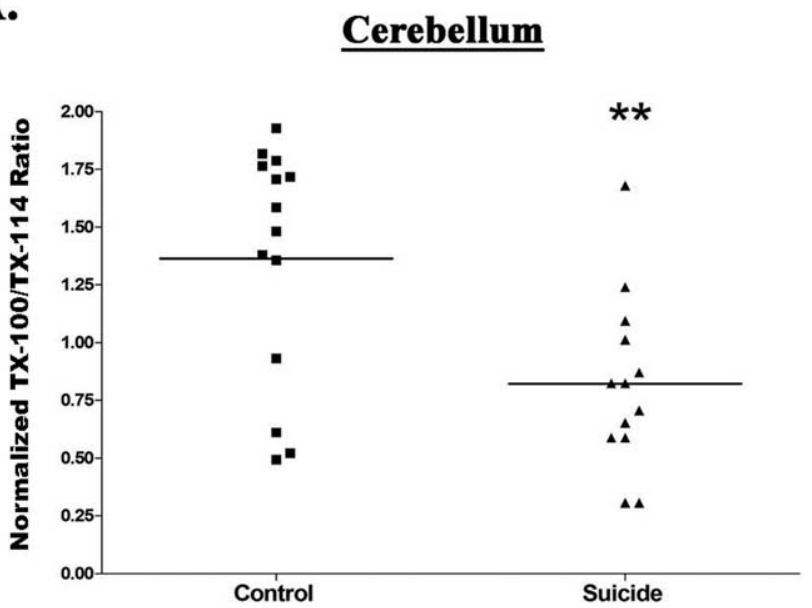

B.

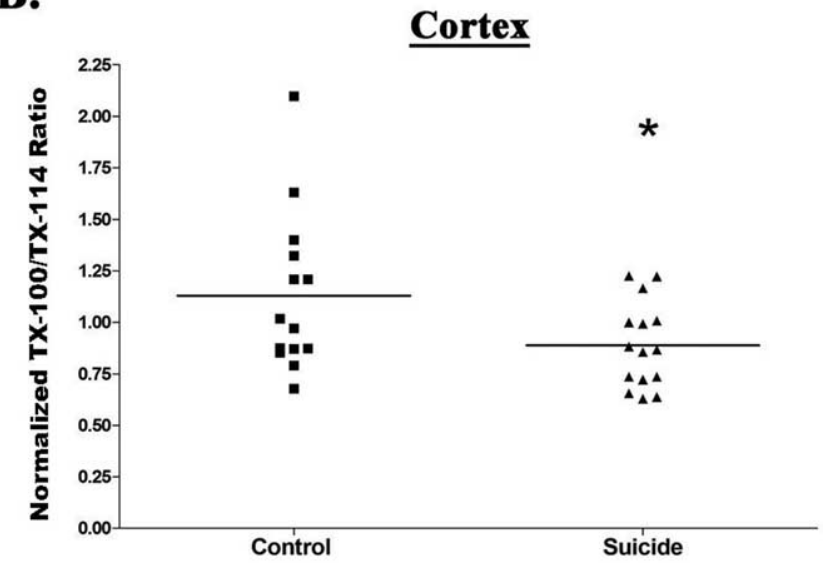

Figure 3. Depressed subjects have significantly more raft-associated Gs $\alpha$ in the cerebellum and prefrontal cortex compared with control subjects. Graphic representation of the normalized densitometric ratios of TX-100-soluble and TX-100-resistant Gs $\alpha$ (TX-100/TX-114). A, Data were acquired from gels and autoradiographs on cerebellum samples. An unpaired $t$ test was performed and the means were found to be significantly different, $p<0.02$ ( $n=14$ for control and $n=13$ for suicide subjects). $\boldsymbol{B}$, Data were acquired from gels and autoradiographs from prefrontal cortex samples, and the average of those ratios is presented. An unpaired $t$ test was performed, and the means were found to be significantly different, $p<0.05$ ( $n=14$ for control and $n=15$ for suicide subjects).

trend in the amount of Gs $\alpha$ localized to each membrane region compared with the localization in the cerebellum (Fig. 2 A). Control subjects have a greater amount of TX-100-soluble (TX-100) Gs $\alpha$ compared with TX-100-resistant (TX-114) Gs $\alpha$, whereas suicide subjects have less TX-100-soluble Gs $\alpha$ compared with TX-100-resistant Gs $\alpha$ in both brain regions (Fig. $2 A, B$ ).

More impressively, examination of the ratio of TX-100- versus TX-114-extractable Gs $\alpha$ reveals a significant difference between suicide and control subjects in both the cerebellum ( $\mathrm{df}=$ $1,24 ; F=8.17 ; p=0.009)$ (Fig. $3 A)$ and the PFC (Fig. $3 B)(\mathrm{df}=$ $1,24 ; F=4.3 ; p<0.05)$. This difference between suicide and control subjects remained significant when individual covariates were used to analyze the data by ANCOVA. For example, the TX-100/TX-114 ratio remained significantly different in the PFC when age $(\mathrm{df}=1,26 ; F=4.12 ; p=0.05)$, gender $(\mathrm{df}=1,26 ; F=$ $5.23 ; p=0.04)$, PMI $(\mathrm{df}=1,26 ; F=4.38 ; p=0.046)$, or $\mathrm{pH}$ of the brain ( $\mathrm{df}=1,26 ; F=5.22 ; p=0.04$ ) were used as covariates (Fig. $3 A$ ). Similarly, ANCOVA showed that the significant difference between suicide subjects and normal controls remained the same in cerebellum when age $(\mathrm{df}=1,24 ; F=9.22 ; p=0.006)$, gender $(\mathrm{df}=1,24 ; F=10.92 ; p=0.003), \mathrm{PMI}(\mathrm{df}=1,24 ; F=10.85 ; p=$ 0.003 ), or $\mathrm{pH}$ of the brain ( $\mathrm{df}=1,24 ; F=8.10 ; p=0.009$ ) was used as covariate (Fig. $3 B$ ). Despite no change in the total amount of membrane extractable Gs $\alpha$ in the cerebellum between both groups, there is a significant increase in the amount of TX-100resistant Gs $\alpha$ in the suicide subjects as demonstrated by the decreased TX-100/TX-114 ratio (Fig. 3A). A similar result was observed in the PFC (Fig. 3B), although not to the extent observed in the cerebellum. It is apparent from data presented in Figures 2 and 3 that less Gs $\alpha$ is available for coupling with adenylyl cyclase in both the cerebellum and PFC of depressed individuals compared with nondepressed controls.

Using positive antidepressant toxicology as a confounding variable, the differences in the TX-100/TX-114 ratio remained significant in the PFC $(\mathrm{df}=1,26 ; F=4.2 ; p=0.04)$ and the cerebellum $(\mathrm{df}=1,26 ; F=13.45 ; p<0.01)$ between control and suicide subjects. It was next determined whether the observed changes in ratio of TX-100/TX-114 in the suicide group were related to a positive antidepressant screen. A comparison of suicide cases who tested positive for antidepressants in the toxicology screen at the time of death $(n=3)$ and those who did not $(n=13)$ showed no significant differences in ratio of TX-100/ TX-114 in both PFC $(t=1.22 ; \mathrm{df}=12 ; p=0.24)$ and cerebellum $(t=2.5 ; \mathrm{df}=13 ; p=0.3)$ between suicide subjects with and without positive antidepressant screen results. It is noteworthy, however, that the three individuals showing the presence of antidepressants were all drug overdose cases and several compounds in addition to antidepressants were present in serum. Thus, it is likely that these subjects ingested antidepressants in the process of completing suicide and were not maintained on these drugs in a therapeutic regimen.

\section{Effects of confounding variables}

The effects of other potential confounding variables, namely, age, $\mathrm{PMI}$, or $\mathrm{pH}$ of the brain were evaluated with respect to ratio of TX-100/TX-114 in PFC and cerebellum. No significant effects of age, PMI, or $\mathrm{pH}$ of the brain were observed, either in PFC or cerebellum. Comparison studies showed no significant differences in any of the measures between males and females in ratio of TX-100/TX-114 in PFC and cerebellum. This relationship was also tested by examining individual values and PMI, and again there was no decrease in Gs $\alpha$ levels with increased PMI. The relationship of PMI with Gs $\alpha$ solubility in the individual TX-100soluble and TX-100-resistant fractions did not reveal significant correlation in PFC (TX-100: $r=0.04, p=0.82$; TX-114: $r=0.03$, $p=0.84$ ) or cerebellum (TX-100: TX-100/TX-114; TX-114: $r=$ $0.26, p=0.19)$. Similarly, the ratios of TX-100/TX-114 were not significantly correlated with PMI in both PFC $(r=0.02 ; p=0.22)$ and cerebellum $(0.06 ; p=0.76)$. These data are substantiated by a study from Li et al. (1995) that demonstrates the stability of Gs $\alpha$ in postmortem human brains to PMI of $21 \mathrm{~h}$ and an age range of 20-100 years.

To examine whether substances of abuse (primarily alcohol and cocaine) had any effect on the ratio of TX-100/TX-114, normal controls and suicide subjects were compared using positive toxicology for substances of abuse as covariate (ANCOVA). Ratios of TX-100/TX-114 remained significantly different in cerebellum $(\mathrm{df}=1,26 ; F=3.8 ; p=0.05)$ and $\mathrm{PFC}(\mathrm{df}=1,26 ; F=9.7$; $p=0.005$ ) between normal controls and suicide subjects regardless of cocaine and/or alcohol. Positive drug toxicology had no significant effect on individual TX-100 and TX-114 values in PFC and the overall differences that were previously observed between normal controls and suicide subjects remained significant (TX- 


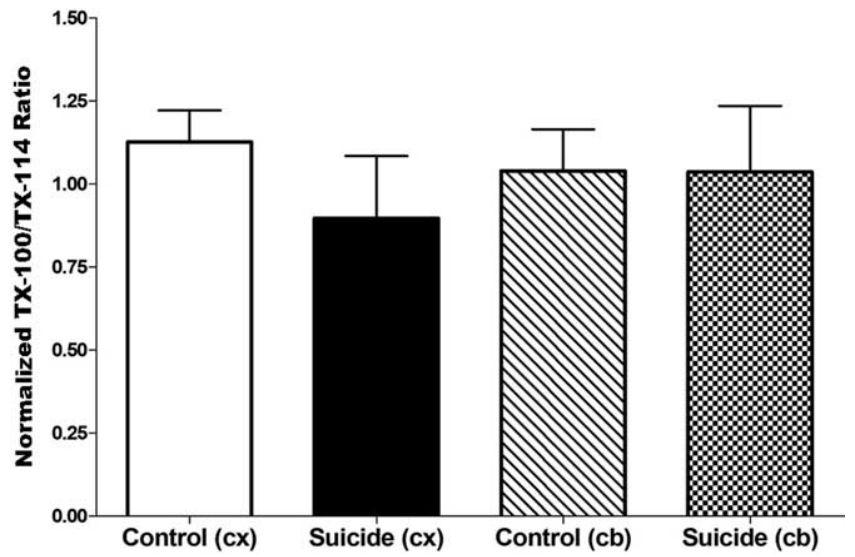

Figure 4. The membrane localization of $\mathrm{Gq} \alpha$ in depressed subjects is unaltered in the prefrontal cortex and cerebellum compared with control subjects. Graphic representation of the normalized densitometric ratios of TX-100-soluble (100) and TX-100-resistant (114) Gq $\alpha$ from a small pool of prefrontal cortex (cx) and cerebellum (cb) samples. Data were acquired from gels and autoradiographs of cerebral cortex and cerebellum samples (some of which involved stripping and reprobing of blots used for $\mathrm{G} s \alpha$ determination). Samples were probed for $\mathrm{Gq} \alpha$. A one-way ANOVA was performed, and no significant differences were found ( $n=4$ for control and $n=4$ for suicide subjects). Error bars indicate SEM.

100: $\mathrm{df}=1,26 ; F=16.5 ; p<0.001 ; \mathrm{TX}-114: \mathrm{df}=1,26 ; F=4.1 ;$ $p=0.05)$.

\section{Gq $\alpha$ membrane localization is unaltered in depressed subjects}

To verify that the alteration in membrane localization was unique to $\mathrm{Gs} \alpha$, another caveolae and lipid raft resident G-protein, Gq $\alpha$ (Oh and Schnitzer, 2001), was examined. In this experiment, the total amount of TX-100- and TX-114-extractable Gq $\alpha$ in cerebellum membranes was unchanged between control and suicide subjects [control $(n=3)$ : mean, $0.9737 \pm 0.1830 \mathrm{AU}$; suicide $(n=3)$ : mean, $1.137 \pm 0.3720 \mathrm{AU} ; p=0.5324]$. Unlike the results with $\mathrm{Gs} \alpha$, there was no significant difference in the total TX-100-soluble and TX-100-resistant Gq $\alpha$ in the PFC between the two groups [control $(n=3)$ : mean, $0.9713 \pm 0.0251 \mathrm{AU}$; suicide $(n=4)$ : mean, $1.038 \pm 0.2140 ; p=0.6212]$. TX-100soluble and TX-100-resistant fractions from samples previously examined for Gs $\alpha$ content were examined for Gq $\alpha$ content by SDS-PAGE followed by Western blotting ( $n=4$ from control and suicide subjects in each brain region). The data in Figure 4 demonstrate that membrane-associated $\mathrm{Gq} \alpha$ is distributed equally between TX-100-soluble and TX-100-resistant membrane domains in both the cerebellum and PFC of suicide and control subjects.

\section{Discussion}

This study demonstrates that functional alterations of Gs $\alpha$ may be attributable to the increased relegation of that protein to lipid raft membrane microdomains in two brain regions of depressed human subjects (summarized in Table 2). This is consistent with a number of cell and animal studies revealing increased coupling between Gs $\alpha$ and adenylyl cyclase subsequent to chronic antidepressant treatment (Ozawa and Rasenick, 1989, 1991; Chen and Rasenick, 1995; Dowlatshahi et al., 1999).

The localization of G-proteins to specific membrane domains such as caveolae and lipid rafts has generated interest as to how these cholesterol and sphingolipid-rich detergent-resistant membrane domains modulate G-protein targeting and function (Bayewitch et al., 2000; Brown and London, 2000; Moffett et al.,
Table 2. Summary of results

\begin{tabular}{lll}
\hline & Cerebellum & PFC \\
\hline Total extractable Gs & NS & $\downarrow$ Suicide \\
TX-100 soluble (TX-100) & NS & $\downarrow$ Suicide \\
TX-100 resistant (TX-114) & NS & $\downarrow$ Suicide \\
ANCOVA (PMI, pH, age) TX-100 & - & No effect \\
ANCOVA (PMI, pH, age) TX-114 & - & No effect \\
ANCOVA (AD) TX-100 & No effect & No effect \\
ANCOVA (AD) TX-114 & No effect & No effect \\
ANCOVA (drug) TX-100 & - & No effect \\
ANCOVA (drug) TX-114 & - & No effect \\
TX-100/TX-114 Ratio & $\downarrow$ Suicide & $\downarrow$ Suicide \\
ANCOVA (PMI, pH, age) TX-100/TX-114 & No effect & No effect \\
ANCOVA (AD) TX-100/TX-114 & No effect & No effect \\
ANCOVA (drug) TX-100/TX-114 & No effect & No effect \\
\hline
\end{tabular}

$\overline{\text { NS, No significant difference between control and suicide subjects; } \downarrow \text { Suicide, significant decrease in the suicide }}$ subjects compared with control; - not measured; ANCOVA, analysis of confounding variables (in parentheses) was used when there was a significant difference between values measured and when antidepressants were found in the subjects at the time of death; no effect, analysis of the data with the confounding variable had no effect on the original analysis.

2000; Ostrom and Insel, 2004; Allen et al., 2005). A recent study by Allen et al. (2005) suggests that $\mathrm{Gs} \alpha$ is targeted to lipid rafts during the process of desensitization, and, as such, lipid rafts represent a membrane domain of diminished Gs $\alpha$-adenylyl cyclase signaling. Conversely, another study has demonstrated that intact caveolae membrane domains are essential for signaling via certain Gq $\alpha$-coupled receptors (Bhatnagar et al., 2004). Thus, lipid raft/caveolae membrane domains appear to be important regulatory domains for G-protein signaling (for review, see Allen et al., 2007).

In this report, we find that $\mathrm{Gs} \alpha$ is localized to both TX-100resistant raft-enriched and TX-100-soluble non-raft membrane domains in the human prefrontal cortex and cerebellum (Fig. 1). Recent studies have demonstrated that Gs $\alpha$ is localized to TX100 -resistant membrane domains and the tips of elongated processes of C6 glioma cells, and this localization is altered after chronic antidepressant treatment (Toki et al., 1999; Donati et al., 2001; Donati and Rasenick, 2005). Similarly, we have observed an altered distribution of Gs $\alpha$ in the TX-100-resistant and the nonraft TX-100-soluble membrane domains in the PFC and cerebellum of depressed suicide subjects compared with control subjects (Figs. 2, 3).

Abnormalities in the cAMP signaling cascade of the human brain from suicide subjects have been studied for over two decades (Cowburn et al., 1994; Pacheco et al., 1996; Dowlatshahi et al., 1999; Stewart et al., 2001; Dwivedi et al., 2002, 2004; Pandey et al., 2005). A consistent finding has been decreased forskolinstimulated adenylyl cyclase activity without a change in expression/concentration of Gs $\alpha$ protein (Cowburn et al., 1994; Dowlatshahi et al., 1999; Stewart et al., 2001), whereas other studies have demonstrated an increase in Gs $\alpha$ protein and mRNA levels in the frontal cortex of subjects with major depression (Pacheco et al., 1996; Dwivedi et al., 2002). The brain regions examined were the temporal and occipital cortex (Dowlatshahi et al., 1999; Stewart et al., 2001) as well as the frontal cortex (Cowburn et al., 1994; Pacheco et al., 1996; Dwivedi et al., 2002). Downstream in the cAMP signaling pathway, decreased PKA (protein kinase A) activity has been observed in the PFC of these same suicide subjects (Dwivedi et al., 2004; Pandey et al., 2005). This is expected in tissue in which cAMP production is suppressed. It is noteworthy in this regard that many studies have used human blood to model the biochemistry of the brain. Studies using human platelets suggest that adenylyl cyclase may, in fact, serve as a biological marker 
for depression (Mooney et al., 1988, 1998; Garcia-Sevilla et al., 1990; Pandey et al., 1990a,b; Menninger and Tabakoff, 1997; Menninger et al., 2000; Hoffman et al., 2002; Hines and Tabakoff, 2005). The most recent of these studies demonstrated markedly lower levels of basal, forskolin-, cesium fluoride-, and $\mathrm{Gpp}(\mathrm{NH}) \mathrm{p}$-stimulated platelet adenylyl cyclase activity in subjects with a history of major depression compared with control subjects (Hines and Tabakoff, 2005). This suggested a possible defect in the ability of Gs $\alpha$ to activate adenylyl cyclase. Consistent with these findings, it has been reported that chronic antidepressant treatment increases the expression and activity of CREB (cAMP response element-binding protein) in the rat brain (Nibuya et al., 1996; Duman et al., 1997; Takahashi et al., 1999; Thome et al., 2000). The finding that overall Gs $\alpha$ is only slightly increased in the PFC of suicide subjects whereas the amount of detergent-extractable Gs $\alpha$ is diminished suggests that studies of effector systems or subcellular localization are more meaningful than those attempting to establish global changes in protein or mRNA.

It was not expected that cerebellum would show an altered localization of $\mathrm{Gs} \alpha$ in depressed subjects similar to that seen in PFC. However, there is behavioral, imaging, and biochemical evidence that the cerebellum may be involved in the etiology of major depression. The cerebellum has neuronal connections to brainstem nuclei that supply the limbic system and PFC with various monoamines, including serotonin and norepinephrine (Schmahmann, 2004). Previous studies of cerebellar atrophy identified patients with intellectual, emotional, and behavioral responses reminiscent of individuals with irreversible character and personality disorders (Schmahmann, 1991). In fact, people with cerebellar lesions have displayed characteristics like passivity, blunting of emotion, and disinhibition of restraint similar to the depressed and manic states characteristic of mood disorders (Schmahmann and Sherman, 1998). Imaging studies suggest that a tonic increase in cerebellar activity is characteristic of major depression as determined by positron emission tomography (Videbech et al., 2001) and glucose utilization studies (Kimbrell et al., 2002). Accordingly, successful treatment of depression with venlafaxine has been associated with decreased blood flow to the cerebellum (Davies et al., 2003).

Dysregulation of the corticotropin-releasing factor (CRF) system has been implicated in the etiology of depression and anxiety (Nemeroff, 1992; Owens and Nemeroff, 1993; Arborelius et al., 1999). This signaling pathway is mediated through the G-protein-coupled receptors, CRF-1 and CRF-2, and this receptor is abundant in both frontal cortex and cerebellum (Kostich et al., 2004). In accordance with these studies, the observed enrichment of Gs $\alpha$ in the TX-100-resistant membrane domains in the cerebellum and PFC of suicide subjects (Figs. 2, 3) may suggest a role in altered CRF-1 signaling. CRF-1 antagonists have demonstrated antidepressant-like activity in animal models (Nielsen, 2006).

Our data clearly demonstrate that, in the cerebellum of depressed suicide subjects, Gs $\alpha$ is preferentially localized to TX100-resistant, lipid raft/cytoskeleton-enriched membrane domains, whereas the control subjects have a greater proportion of nonraft TX-100-soluble Gs $\alpha$ (Figs. 2, 3; Table 2). These results are mirrored in the PFC of the same subjects, although to a lesser, yet still significant degree (Figs. 2, 3; Table 2). The TX-100soluble Gs $\alpha$ is more likely to interact with adenylyl cyclase because the TX-100-resistant membrane regions serve as inhibitory domains for Gs $\alpha$ signaling (Li et al., 1995) and they are much more rigid cholesterol and sphingolipid-rich membrane structures (Brown and London, 2000).

In a live cell study, we demonstrated that Gs $\alpha$ is internalized through lipid rafts, and that this internalized Gs $\alpha$ is not activating adenylyl cyclase (Allen et al., 2005). Rybin et al. (2000) have demonstrated that cholesterol depletion increases adenylyl cyclase signaling in cardiac myocytes, and we have recently seen the same phenomenon in C6 glioma cells in which cholesterol was modified or in which lipid rafts/caveolae were disrupted genetically (J. A. Allen, B. L. Roth, and M. M. Rasenick, unpublished observations). Curiously, similar studies with the Gq-coupled 5- $\mathrm{HT}_{2 \mathrm{~A}}$ and PAR-1 thrombin receptor reveal a dependence on intact caveolae for proper Gq signaling (Bhatnagar et al., 2004). This suggests that, rather than a wholesale disruption of caveolae or lipid rafts, some specific lipid raft anchor of Gs $\alpha$ is modified in depression or by antidepressant treatment.

Previous studies in both rats and C6 glioma cells showed that chronic antidepressant treatment liberates Gs $\alpha$ from the inhibitory TX-100-resistant membrane domains (Toki et al., 1999; Donati et al., 2001; Donati and Rasenick, 2005) as well as increases its association with adenylyl cyclase (Chen and Rasenick, 1995; Toki et al., 1999). Gi $\alpha$ and Go $\alpha$ were unaffected in these studies. Together, these observations demonstrate that the increased localization of Gs $\alpha$ in the TX-100-resistant membrane domains of depressed individuals may prevent Gs $\alpha$ from associating with and activating adenylyl cyclase, thus preventing the propagation of the cAMP signal. These findings are supported by recent evidence suggesting that antidepressant drugs concentrate in raftlike plasma membrane domains (Eisensamer et al., 2005). The intercalation of these drugs may physically inhibit the localization of Gs $\alpha$ to the raft domains. Thus, it appears that antidepressants may exert their observed effects on cAMP signaling by liberating Gs $\alpha$ from TX-100-resistant membrane domains, where it accumulates during the course of depression. Furthermore, the ratio of TX-100-soluble to TX-100-resistant Gs $\alpha$ may prove to be a useful biomarker for human depression and response to antidepressant therapy.

\section{References}

Allen JA, Yu JZ, Donati RJ, Rasenick MM (2005) Beta-adrenergic receptor stimulation promotes $\mathrm{G}$ alpha s internalization through lipid rafts: a study in living cells. Mol Pharmacol 67:1493-1504.

Allen JA, Halverson-Tamboli RA, Rasenick MM (2007) Lipid raft microdomains and neurotransmitter signaling. Nat Rev Neurosci 8:128-140.

Arborelius L, Owens MJ, Plotsky PM, Nemeroff CB (1999) The role of corticotropin-releasing factor in depression and anxiety disorders. J Endocrinol 160:1-12.

Bayewitch ML, Nevo I, Avidor-Reiss T, Levy R, Simonds WF, Vogel Z (2000) Alterations in detergent solubility of heterotrimeric $G$ proteins after chronic activation of $\mathrm{G}(\mathrm{i} / \mathrm{o})$-coupled receptors: changes in detergent solubility are in correlation with onset of adenylyl cyclase superactivation. Mol Pharmacol 57:820-825.

Bhatnagar A, Sheffler DJ, Kroeze WK, Compton-Toth B, Roth BL (2004) Caveolin-1 interacts with 5-HT2A serotonin receptors and profoundly modulates the signaling of selected Galphaq-coupled protein receptors. J Biol Chem 279:34614-34623.

Brown DA, London E (2000) Structure and function of sphingolipid- and cholesterol-rich membrane rafts. J Biol Chem 275:17221-17224.

Chen J, Rasenick MM (1995) Chronic treatment of C6 glioma cells with antidepressant increases functional coupling between a $\mathrm{G}$ protein (Gs) and adenylyl cyclase. J Neurochem 64:724-732.

Cowburn RF, Marcusson JO, Eriksson A, Wiehager B, O’Neill C (1994) Adenylyl cyclase activity and G-protein subunit levels in postmortem frontal cortex of suicide victims. Brain Res 633:297-304.

Davies J, Lloyd KR, Jones IK, Barnes A, Pilowsky LS (2003) Changes in regional cerebral blood flow with venlafaxine in the treatment of major depression. Am J Psychiatry 160:374-376. 
De Montis GM, Devoto P, Gessa GL, Porcella A, Serra G, Tagliamonte A (1990) Selective adenylate cyclase increase in the limbic area of long-term imipramine-treated rats. Eur J Pharmacol 180:169-174.

Donati RJ, Rasenick MM (2003) G protein signaling and the molecular basis of antidepressant action. Life Sci 73:1-17.

Donati RJ, Rasenick MM (2005) Chronic antidepressant treatment prevents accumulation of gsalpha in cholesterol-rich, cytoskeletal-associated, plasma membrane domains (lipid rafts). Neuropsychopharmacology 30:1238-1245.

Donati RJ, Thukral C, Rasenick MM (2001) Chronic treatment of C6 glioma cells with antidepressant drugs results in a redistribution of Gsalpha. Mol Pharmacol 59:1426-1432.

Dowlatshahi D, MacQueen GM, Wang JF, Reiach JS, Young LT (1999) G protein-coupled cyclic AMP signaling in postmortem brain of subjects with mood disorders: effects of diagnosis, suicide, and treatment at the time of death. J Neurochem 73:1121-1126.

Drevets WC (2001) Neuroimaging and neuropathological studies of depression: implications for the cognitive-emotional features of mood disorders. Curr Opin Neurobiol 11:240-249.

Duman RS, Heninger GR, Nestler EJ (1997) A molecular and cellular theory of depression. Arch Gen Psychiatry 54:597-606.

Dwivedi Y, Rizavi HS, Conley RR, Roberts RC, Tamminga CA, Pandey GN (2002) mRNA and protein expression of selective alpha subunits of G proteins are abnormal in prefrontal cortex of suicide victims. Neuropsychopharmacology 27:499-517.

Dwivedi Y, Rizavi HS, Shukla PK, Lyons J, Faludi G, Palkovits M, Sarosi A, Conley RR, Roberts RC, Tamminga CA, Pandey GN (2004) Protein kinase A in postmortem brain of depressed suicide victims: altered expression of specific regulatory and catalytic subunits. Biol Psychiatry 55:234-243.

Eisensamer B, Rammes G, Gimpl G, Shapa M, Ferrari U, Hapfelmeier G, Bondy B, Parsons C, Gilling K, Zieglgansberger W, Holsboer F, Rupprecht R (2003) Antidepressants are functional antagonists at the serotonin type 3 (5-HT3) receptor. Mol Psychiatry 8:994-1007.

Eisensamer B, Uhr M, Meyr S, Gimpl G, Deiml T, Rammes G, Lambert JJ, Zieglgansberger W, Holsboer F, Rupprecht R (2005) Antidepressants and antipsychotic drugs colocalize with 5-HT3 receptors in raft-like domains. J Neurosci 25:10198-10206.

Garcia-Sevilla JA, Padro D, Giralt MT, Guimon J, Areso P (1990) Alpha 2-adrenoceptor-mediated inhibition of platelet adenylate cyclase and induction of aggregation in major depression. Effect of long-term cyclic antidepressant drug treatment. Arch Gen Psychiatry 47:125-132.

Harrison PJ, Heath PR, Eastwood SL, Burnet PW, McDonald B, Pearson RC (1995) The relative importance of premortem acidosis and postmortem interval for human brain gene expression studies: selective mRNA vulnerability and comparison with their encoded proteins. Neurosci Lett 200:151-154.

Hines LM, Tabakoff B (2005) Platelet adenylyl cyclase activity: a biological marker for major depression and recent drug use. Biol Psychiatry 58:955-962.

Hoffman PL, Glanz J, Tabakoff B (2002) Platelet adenylyl cyclase activity as a state or trait marker in alcohol dependence: results of the WHO/ISBRA Study on State and Trait Markers of Alcohol Use and Dependence. Alcohol Clin Exp Res 26:1078-1087.

Holsboer F (2000) The corticosteroid receptor hypothesis of depression. Neuropsychopharmacology 23:477-501.

Kamada H, Saito T, Hatta S, Toki S, Ozawa H, Watanabe M, Takahata N (1999) Alterations of tubulin function caused by chronic antidepressant treatment in rat brain. Cell Mol Neurobiol 19:109-117.

Kimbrell TA, Ketter TA, George MS, Little JT, Benson BE, Willis MW, Herscovitch P, Post RM (2002) Regional cerebral glucose utilization in patients with a range of severities of unipolar depression. Biol Psychiatry 51:237-252.

Kostich WA, Grzanna R, Lu NZ, Largent BL (2004) Immunohistochemical visualization of corticotropin-releasing factor type 1 (CRF1) receptors in monkey brain. J Comp Neurol 478:111-125.

Li S, Okamoto T, Chun M, Sarguacini M, Casanova JE, Hansen SH, Nishimoto I, Lisanti MP (1995) Evidence for a regulated interaction between heterotrimeric G proteins and caveolin. J Biol Chem 270:15693-15701.

Mayberg HS (2003) Positron emission tomography imaging in depression: a neural systems perspective. Neuroimaging Clin N Am 13:805-815.

Meltzer HY, Fang VS, Tricou BJ, Robertson A (1982) Effect of antidepres- sants on neuroendocrine axis in humans. Adv Biochem Psychopharmaco 32:303-316.

Menkes D, Rasenick MM, Wheeler M, Bitensky MW (1983) Guanosine triphosphate activation of brain adenylate cyclase: enhancement by longterm antidepressant treatment. Science 219:65-67.

Menninger JA, Tabakoff B (1997) Forskolin-stimulated platelet adenylyl cyclase activity is lower in persons with major depression. Biol Psychiatry 42:30-38.

Menninger JA, Baron AE, Conigrave KM, Whitfield JB, Saunders JB, Helander A, Eriksson CJ, Grant B, Hoffman PL, Tabakoff B (2000) Platelet adenylyl cyclase activity as a trait marker of alcohol dependence. WHO/ ISBRA Collaborative Study Investigators. International Society for Biomedical Res on Alcoholism. Alcohol Clin Exp Res 24:810-821.

Moffett S, Brown DA, Linder ME (2000) Lipid-dependent targeting of G proteins into rafts. J Biol Chem 275:2191-2198.

Mooney JJ, Schatzberg AF, Cole JO, Kizuka PP, Salomon M, Lerbinger J, Pappalardo KM, Gerson B, Schildkraut JJ (1988) Rapid antidepressant response to alprazolam in depressed patients with high catecholamine output and heterologous desensitization of platelet adenylate cyclase. Biol Psychiatry 23:543-559.

Mooney JJ, Samson JA, McHale NL, Colodzin R, Alpert J, Koutsos M, Schildkraut JJ (1998) Signal transduction by platelet adenylate cyclase: alterations in depressed patients may reflect impairment in the coordinated integration of cellular signals (coincidence detection). Biol Psychiatry 43:574-583.

Nemeroff CB (1992) New vistas in neuropeptide research in neuropsychiatry: focus on corticotropin-releasing factor. Neuropsychopharmacology 6:69-75.

Nibuya M, Nestler E, Duman R (1996) Chronic antidepressant administration increases the expression of cAMP response element binding protein in the rat hippocampus. J Neurosci 16:2365-2372.

Nielsen DM (2006) Corticotropin-releasing factor type-1 receptor antagonists: the next class of antidepressants? Life Sci 78:909-919.

Oh P, Schnitzer JE (2001) Segregation of heterotrimeric G proteins in cell surface microdomains. G(q) binds caveolin to concentrate in caveolae, whereas $\mathrm{G}(\mathrm{i})$ and $\mathrm{G}(\mathrm{s})$ target lipid rafts by default. Mol Biol Cell 12:685-698.

Ostrom RS, Insel PA (2004) The evolving role of lipid rafts and caveolae in G protein-coupled receptor signaling: implications for molecular pharmacology. Br J Pharmacol 143:235-245.

Owens MJ, Nemeroff CB (1993) The role of corticotropin-releasing factor in the pathophysiology of affective and anxiety disorders: laboratory and clinical studies. Ciba Found Symp 172:296-316.

Owens MJ, Nemeroff CB (1999) Corticotropin-releasing factor antagonists in affective disorders. Expert Opin Investig Drugs 8:1849-1858.

Ozawa H, Rasenick MM (1989) Coupling of the stimulatory GTP-binding protein Gs to rat synaptic membrane adenylate cyclase is enhanced subsequent to chronic antidepressant treatment. Mol Pharmacol 36:803-808.

Ozawa H, Rasenick MM (1991) Chronic electroconvulsive treatment augments coupling of the GTP-binding protein Gs to the catalytic moiety of adenylyl cyclase in a manner similar to that seen with chronic antidepressant drugs. J Neurochem 30:330-338.

Pacheco MA, Stockmeier C, Meltzer HY, Overholser JC, Dilley GE, Jope RS (1996) Alterations in phosphoinositide signaling and G-protein levels in depressed suicide brain. Brain Res 723:37-45.

Pandey GN, Pandey SC, Davis JM (1990a) Peripheral adrenergic receptors in affective illness and schizophrenia. Pharmacol Toxicol 66 [Suppl 3]:13-36.

Pandey GN, Pandey SC, Janicak PG, Marks RC, Davis JM (1990b) Platelet serotonin-2 receptor binding sites in depression and suicide. Biol Psychiatry 28:215-222.

Pandey GN, Dwivedi Y, Ren X, Rizavi HS, Mondal AC, Shukla PK, Conley RR (2005) Brain region specific alterations in the protein and mRNA levels of protein kinase A subunits in the post-mortem brain of teenage suicide victims. Neuropsychopharmacology 30:1548-1556.

Perez J, Tinelli D, Brunello N, Racagni G (1989) cAMP-dependent phosphorylation of soluble and crude microtubule fractions of rat cerebral cortex after prolonged desmethylimipramine treatment. Eur J Pharmacol 172:305-316.

Perez J, Tinelli D, Bianchi E, Brunello N, Racagni G (1991) cAMP binding proteins in the rat cerebral cortex after administration of selective 5-HT 
and NE reuptake blockers with antidepressant activity. Neuropsychopharmacology 4:57-64.

Rybin VO, Xu X, Lisanti MP, Steinberg SF (2000) Differential targeting of beta-adrenergic receptor subtypes and adenylyl cyclase to cardiomyocyte caveolae. A mechanism to functionally regulate the cAMP signaling pathway. J Biol Chem 275:41447-41457.

Schmahmann JD (1991) An emerging concept. The cerebellar contribution to higher function. Arch Neurol 48:1178-1187.

Schmahmann JD (2004) Disorders of the cerebellum: ataxia, dysmetria of thought, and the cerebellar cognitive affective syndrome. J Neuropsychiatry Clin Neurosci 16:367-378.

Schmahmann JD, Sherman JC (1998) The cerebellar cognitive affective syndrome. Brain 121:561-579.

Spitzer RL, Williams JB, Gibbon M, First MB (1992) The Structured Clinical Interview for DSM-III-R (SCID). I: History, rationale, and description. Arch Gen Psychiatry 49:624-629.

Stewart RJ, Chen B, Dowlatshahi D, MacQueen GM, Young LT (2001) Abnormalities in the cAMP signaling pathway in post-mortem brain tissue from the Stanley Neuropathology Consortium. Brain Res Bull 55:625-629.
Takahashi M, Terwilliger R, Lane C, Mezes PS, Conti M, Duman RS (1999) Chronic antidepressant administration increases the expression of cAMP-specific phosphodiesterase $4 \mathrm{~A}$ and $4 \mathrm{~B}$ isoforms. J Neurosci 19:610-618.

Thome J, Sakai N, Shin K, Steffen C, Zhang YJ, Impey S, Storm D, Duman RS (2000) cAMP response element-mediated gene transcription is upregulated by chronic antidepressant treatment. J Neurosci 20:4030-4036.

Toki S, Donati RJ, Rasenick MM (1999) Treatment of C6 glioma cells and rats with antidepressant drugs increases the detergent extraction of $\mathrm{G}$ (s alpha) from plasma membrane. J Neurochem 73:1114-1120.

Valentino RJ, Curtis AL (1991) Antidepressant interactions with corticotropin-releasing factor in the noradrenergic nucleus locus coeruleus. Psychopharmacol Bull 27:263-269.

Videbech P, Ravnkilde B, Pedersen AR, Egander A, Landbo B, Rasmussen NA, Andersen F, Stodkilde-Jorgensen H, Gjedde A, Rosenberg R (2001) The Danish PET/depression project: PET findings in patients with major depression. Psychol Med 31:1147-1158.

Zalcman S, Endicott J (1983) Diagnostic evaluation after death. Rockville, MD: National Institute of Mental Health, Neuroscience Research Branch. 